What is the importance and implication of fundamental constants and SI units in physics and technology? This question is topical as considerable progress has been made recently in the precision measurement of fundamental constants, especially through quantum phenomena on a macroscopic scale such as the von Klitzing effect and the AC Josephson effect. This relates also to the world-wide efforts to attain consistent sets of units and physical constants of nature, which could lead to a future system of units, completely quantum effect related.

In the past century man discovered the key to the natural sciences and technology, and triggered a unique economic development. Production and trade was at first still restricted to small market areas, but it steadily expanded into a world trade extending beyond local boundaries. At the same time, efforts were made towards the standardization of measures, which today have ended in the creation and world-wide application of the SI units. The most important step was taken already during the French revolution. About 100 years later, in 1875, an international metrological agreement, the "International Meter Convention" was concluded whereby 17 signatory states bound themselves to introduce the "metric system" officially in their countries and to effect its application and dissemination. The People's Republic of China joined the Meter Convention in 1977 as the 45 th member state. Today, metric units are obligatory in more than 100 states.

\section{From Prototype to Quantum Measures}

Before discussing the current situation and its relation to fundamental constants, a clear delimitation of the terms definition, realization, and reproduction of units is necessary. The international system of units elaborated by the Meter Convention is coherent; i.e., besides the seven base units: second (s), meter (m), kilogram (kg), ampere (A), kelvin (K), mole (mol), candela (cd), it comprises only those derived units that can be formed from the base units as power products with integral exponents and no factor differing from unity. For example: $1 \mathrm{~V}=1 \mathrm{~s}^{-3} \mathrm{~m}^{2} \mathrm{~kg} \mathrm{~A}^{-1}$

is valid for the unit of voltage and $1 \Omega=1 \mathrm{~s}^{-3} \mathrm{~m}^{2} \mathrm{~kg} \mathrm{~A}^{-2}$

for that of electrical resistance.

The definitions of the units within the $\mathrm{SI}$ are based on ideal conditions with an uncertainty of zero assigned to them. They must be distinguished from their realizations, that is their embodiments in the laboratory which must be kept within certain limits of uncertainty, necessa-

Fundamental Constants and

\title{
SI* units
}

\author{
Volkar Kose, Braunschweig \\ (Electrical Science Division, Physikalisch-Technische Bundesanstalt)
}

\section{* Système International d'Unités}

rily finite. An almost 100 year-old exception is the still binding definition of the unit of mass $(\mathrm{kg})$ which reads:

"The kilogram is the unit of mass; it is equal to the mass of the international prototype of the kilogram (1889)"

It is an exception as the embodiment in a single prototype itself serves as definition. Consequently, variations in the mass of the kilogram prototype through gas adsorption, which are indeed observed, imply variations in the unit of mass. This is certainly a rather unsatisfactory situation. The problem was already recognized by James Clerk Maxwell in 1870 when he noted on the occasion of a meeting of the British Association for the Advancement of Science:

"If we wish to obtain standards of length, time and mass which shall be absolutely permanent, we must seek them not in the dimensions or the motion, or the mass of our planet, but in the wavelength, the period of vibration, and the absolute mass of these imperishable and unalterable and perfectly similar molecules."

Generalizing his idea means that physical quantities present in the "macrocosm" should be linked to ideal units of the "microcosm" offering the following advantages. They are, when cosmological variations are ignored,

— imperishable

- invariable with regard to time and place

- identical; requiring no prototype and so no hierarchy of standards

- available in any number at any place, and at any time.

However, it took more than one hundred years before the two base units; second (1967) and meter (1960, 1983) were

Table 1

\begin{tabular}{|l|c|c|c|c|c|c|c|}
\hline Base unit & $\mathrm{s}^{*}$ & $\mathrm{~m}^{*}$ & $\mathrm{~kg}$ & $\mathrm{~A}$ & $\mathrm{~K}$ & $\mathrm{~mol}$ & $\mathrm{~cd}$ \\
\hline $\begin{array}{l}\text { Relative } \\
\text { uncertainty } u\end{array}$ & $10^{-14}$ & $10^{-10}$ & 0 & $6 \times 10^{-6}$ & $10^{-4}$ & $10^{-6}$ & $5 \times 10^{-3}$ \\
\hline
\end{tabular}

* Units based on quantum measures defined in Maxwell's sense. The actual definitions decided by the General Conference of the Meter Convention read:

"The second is the duration of

9192631770 periods of the radiation corresponding to the transition between the two hyperfine levels of the ground state of the caesium-133 atom" (1967)

"The meter is the length of the path travelled by light in vacuum in the time interval $1 / 299792458$ of a second" (1983)

By this the meter is metrologically related to the second by a fixed value of the speed of light in vacuum.

The special numbers which appear in the texts are a consequence of the intention to maintain continuity as closely as possible in the size of the unit based on the former prototypes, the platinumiridium meter bar and ephemeris time.

\section{Reproduction of Units via Fundamental Constants}

The realization of the physical units and making them continuously available are two of the main tasks of the metrological institutes in the various countries. In principle, the realization, the reproduction, maintenance and dissemination of a unit are only feasible to a certain experimental uncertainty. For the base units, Table 1 indicates the best relative uncertainties $u$ which are today attainable.

The kilogram prototype is maintained at the Bureau International des Poids et Mesures in Sèvres, Paris and per definitionem as already noted has zero uncertainty. Several countries obtained copies of the prototype which have by balance comparisons, typical relative uncertainties of 1 part in $10^{8}$. The uncertainty of 
the Kelvin is related to a nominal value at room temperature.

It is a general experience that the procedures for the realization of the base as well as the derived units involve extensive metrological work; they are extremely difficult to carry out and are very time consuming. Consequently, they are repeated only about once every decade. On the other hand, the metrological institutes are obliged to disseminate the units within the framework of legal and industrial metrology at any time. Longterm drifts of the standards for dissemination require reproduction of the asmaintained units at intervals of about 0.5 to 1 year.

Max Planck, the founder of quantum physics, stated in 1899 that a physical system of units should not be based on atoms or molecules, but should be founded on more deep-seated reality, the fundamental constants of nature itself. Such natural constants as Planck's constant $h$, the speed of light in vacuum $c$, Boltzmann's constant $k$ or the elementary charge $e$, are universal because of their independence of special substances or atoms. In addition, by using fundamental constants in conjunction with quantum effects, independence of space and time is guaranteed. Such effects on a macroscopic scale can therefore be used for the reproduction of the as-maintained units. Table 2 indicates this situation for the ampere and the derived electrical units:

Fig. 1 demonstrates how vital fundamental constants are for the precise reproduction of units of various physical quantities. A few of the listed procedures were developed about 25 years ago, whereas others are very recent achievements. Klaus von Klitzing ${ }^{2}$ ) discovered in 1980 a quantum effect for the precise determination of the fine structure cons$\tan t \alpha$. The impact of this effect is twofold:

Primarily, there is a fundamental constant aspect which will be discussed later. Secondly, there exists a metrological aspect, because the von Klitzing effect can be used for monitoring the ohm as-maintained (see Fig. 1). We should note that the ohm based on this effect would be a unit in Planck's sense, whereas, for example, the second and the meter are units in Maxwell's sense.

The theory of the von Klitzing effect is not so far advanced as to predict or exclude possible influences which may call in question the validity of the simple relation between $h / e^{2}$ and the observed steps in the Hall resistance. However, right now we have experimental evidence that within 3 parts in $10^{8}, R$ is in-

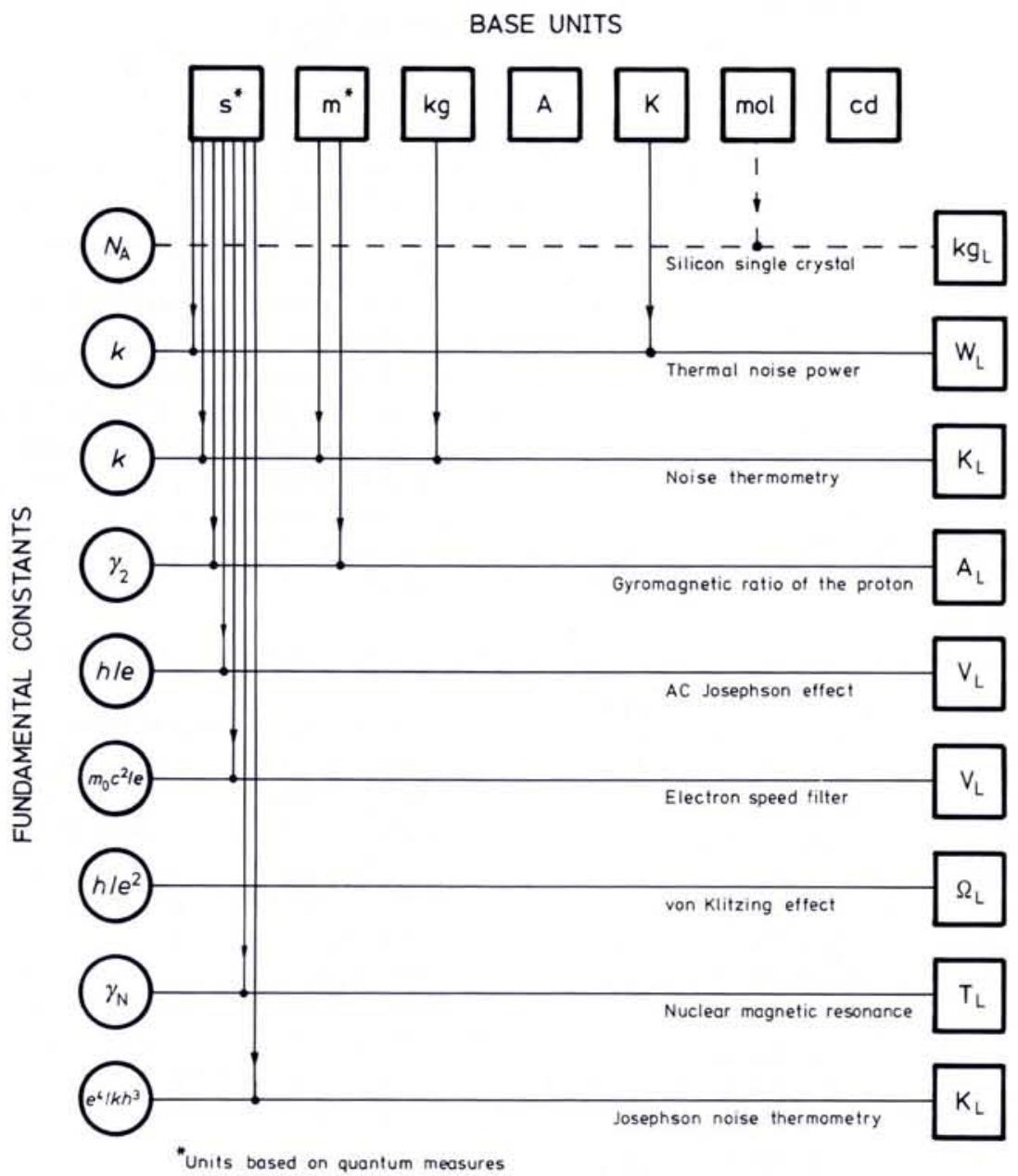

Fig. 1 - Reproduction of units maintained in the laboratory (subscript ' $L$ ") via fundamental constants:

$N_{A}$ Avogadro constant; $k$ Boltzmann constant; $\gamma$, gyromagnetic ratio of the proton; $h$ Planck constant; e elementary charge; $m_{0}$ rest mass of the electron; $\gamma_{N}$ gyromagnetic ratio of certain nuclides. The dashed line means planning stage.

dependent of material (Si MOSFET, GaAs-heterostructure), temperature (1 to $4.2 \mathrm{~K})$, magnetic flux density (7 T; 13 T), and step number $n(2 \text { and } 4)^{3}$ ). Much theoretical and experimental work has still to be done in order to guarantee that this most interesting quantum phenomenon can be used as a world-wide basis for reproducing the ohm, the unit of electrical resistance. In 1986 the Comité Consultatif d'Electricité (CCE) of the International Meter Convention will meet and evaluate the results obtained

Table 2

\begin{tabular}{|c|c|c|c|}
\hline & Ampere & Volt & Ohm \\
\hline Definition & $1 \mathrm{~A}$ & $1 \mathrm{~V}=1 \mathrm{~s}^{-3} \mathrm{~m}^{2} \mathrm{~kg} \mathrm{~A}^{-1}$ & $1 \Omega=1 \mathrm{~s}^{-3} \mathrm{~m}^{2} \mathrm{~kg} \mathrm{~A}^{-2}$ \\
\hline Realization & current balance & voltage balance & $\begin{array}{l}\text { calculable cross } \\
\text { capacitance }\end{array}$ \\
\hline Reproduction & $\begin{array}{l}\text { gyromagnetic ratio } \\
\text { of the proton: } \\
I=\text { const } \times f / \gamma_{1}\end{array}$ & $\begin{array}{l}\text { Josephson effect: } \\
\text { voltage } U=h f / 2 e\end{array}$ & $\begin{array}{l}\text { von Klitzing effect: } \\
\text { quantized Hall } \\
\text { resistance } R=h / e^{2}\end{array}$ \\
\hline $\begin{array}{l}\text { Maintenance } \\
\text { and } \\
\text { Dissemination }\end{array}$ & $\begin{array}{l}\text { standard cell } \\
\text { and } \\
\text { standard resistance }\end{array}$ & $\begin{array}{l}\text { electrochemical } \\
\text { standard cell }\end{array}$ & $\begin{array}{l}\text { wire-based standard } \\
\text { resistance }\end{array}$ \\
\hline
\end{tabular}

in the different countries; it intends to recommend and adopt a unique value of $h / e^{2}$ for international application in precision electrical measurements.

This process is quite analogous to the reproduction of the volt based on the $A C$ Josephson effect. In 1972, the CCE recommended for metrological application a certain number for $h / 2 e$, which was thought to be equal to its SI value within the relative uncertainty of 5 parts in $10^{7}$. Today, from voltage and current balance experiments and interrelations 


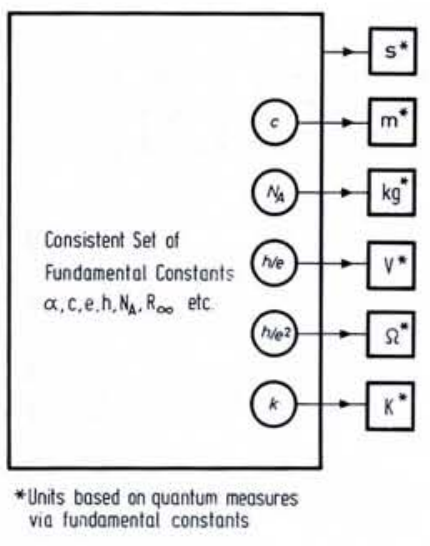

Fig. 2 - Conceivable future system of units based on a consistent set of fundamental constants.

between fundamental constants, we have evidence that the assumed value seems to be incorrect by a few parts in $10^{6}$. As for $h / e^{2}$, in 1986 the CCE intends to adopt a new $h / 2 e$ value on a sound basis of consistent experimental results. Most recently a Josephson junction array with almost 1500 elements in series giving a total quantized voltage of $1 \mathrm{~V}$ at zero current has been successfully developed ${ }^{4}$ ). The cryogenic voltage standards used so far exhibit Josephson voltages in the order of a few millivolts. It is conceivable that recent progress could in the near future lead to the reproduction of the volt to 1 part in $10^{10}$ through the complete elimination of any voltage divider. In addition, it will stimulate Josephson potentiometry in the area of precision electrical metrology.

\section{From Fundamental Constants to SI Units}

There are two ways to realize SI units. Besides the well known direct realizations, there exist indirect methods based on fundamental constants. Sometimes, it is not necessary to know the constants of nature entirely in SI values, only in arbitrary units, say as-maintained units. For instance the ampere $\mathrm{A}$ and the ohm $\Omega{ }^{5}$ ) can be derived in SI units with the following relations, where braces mean numerical values of the quantities:

$$
\begin{gathered}
1 \mathrm{~A}=1 \mathrm{~A}_{\mathrm{L}}\left(\left\{\gamma_{1}\right\}_{\mathrm{L}} /\left\{\gamma_{2}\right\}_{\mathrm{L}}\right)^{1 / 2} \\
1 \Omega=\Omega_{\mathrm{L}}\left(\frac{16\left\{R_{\infty}\right\}}{\left\{\mu_{O}\right\}^{2}\{c\}^{3}} \frac{\mu_{\mathrm{B}}}{\mu_{\mathrm{p}}}\right. \\
\left.\left\{h / e^{2}\right\}_{\mathrm{L}}^{2}\{h / 2 e\}_{\mathrm{L}}\left\{\gamma_{2}\right\}_{\mathrm{L}}\right)^{1 / 3}
\end{gathered}
$$

On the right-hand side of these equations the subscript $L$ refers to the electrical as-maintained units, whereas all mechanical units have to be expressed in SI units. $\left\{\gamma_{2}\right\}_{1}$ stands for the value of the precision measurement of the proton's gyromagnetic ratio in the so-called 'low magnetic field experiment', whereas $\left\{\gamma_{1}\right\}_{\llcorner}$denotes that in the 'high field' one in which a meaningful force measurement is feasible, $\mu_{0}$ is the magnetic constant, $R_{\infty}$ the Rydberg constant, and $\mu_{\mathrm{p}} / \mu_{\mathrm{B}}$ is the ratio of the magnetic moment of the proton to the Bohr magneton.

This way through fundamental constants of realizing the ampere or the ohm, demonstrates an important alternative to the classical procedures as the current balance and the calculable cross capacitance method. However, in both cases the total uncertainty cannot be kept below that of the mechanical SI units involved.

\section{Consistent Set of Fundamental Con- stants}

The fundamental constants listed in Fig. 1 are only those which are relevant for the reproduction of as-maintained units. However, there are several other constants of nature appearing in different branches of physics such as quantum mechanics, quantum electrodynamics, theory of relativity, etc. Together these establish a set of constants which are related to one another by exact theories of physics. One example is the relation of the fine structure constant $\alpha$ to $h / e^{2}$ :

$$
\alpha^{-1}=\frac{2}{\left\{\mu_{0}\right\}\{c\}}\left\{\frac{h}{e^{2}}\right\}\left\{\begin{array}{l}
\Omega_{L} \\
\{\Omega
\end{array}\right\}
$$

$\mu_{\mathrm{o}}$ and $c$ are exactly known in the SI by definition of the ampere and the meter respectively. Eq. 3 demonstrates that a two-dimensional electron gas can contribute to the interaction of light with matter! The von Klitzing effect requires precise knowledge of the quantized Hall resistance (QHR) in SI units, which is at present available with the calculable cross capacitance method to only about 1 part in $10^{7}$. Recent measurements have shown that $\left(h / 2 e^{2}\right)$ can be reproduced to 1 part in $10^{86}$ ) and it is apparent that a better SI ohm is urgently needed to improve the fine structure constant.

However, by replacing $\Omega_{L} / \Omega$ in eq. (3) with eq. (2), the fine structure constant can be related to completely different constants from other areas of physics such as superconductivity. As different experiments are involved, the equations relating the various fundamental constants may yield two different values for $\alpha$. A similar situation exists for several other constants. To be able to specify only one value for each fundamental constant, by applying the least-squares adjustment, a best value of each constant is determined which entails minimum infringement of all interrelations. In this way a consistent set of fundamental constants in physics is obtained. Thus it becomes understandable that this consistency depends very much on correct theoretical models and on the "metrological art" of having no systematic errors in the experiments. Vice versa, any inconsistency in the set of constants can be an indication of either false experiments or incorrect theoretical models. The last least-squares adjustment was published in $1973^{7}$ ), the next is to be expected this year.

\section{System of Units Based on the Set of Fundamental Constants?}

The main objection to the present International System of Units, SI, is the obvious defect that not all units can be traced back with the required precision to quantum measures or fundamental constants. Although the second, the meter, the volt and the ohm could be based with sufficient accuracy on quantum phenomena in Maxwell's or Planck's sense, this does not hold at present for the kilogram. If this problem could be overcome in the future through a better knowledge of the Avogadro constant, one could envisage a system of physical units based on a consistent set of fundamental constants, as indicated in Fig. 2 , where only a few of these constants have to be defined numerically. The consistency of this set would also guarantee the consistency of the physical units.

On the other hand, new physical phenomena can be discovered which can modify our ideas. One recent example was the von Klitzing effect, which opened a completely new door in physics, stimulating the fundamental constants scene and with it, the field of physical units in a completely unforeseen way. We are ready for the next surprise!

\section{REFERENCES}

1. Kose V., 'Basis of Precision Electrical Metrology', Quantum Metrology and Fundamental PhysicalConstants, Ed.P.H. Cutler and A.A. Lucas, Nato ASISeries B, 98 (1983) pp. 209-220.

2. Von Klitzing K., Dorda G. and Pepper M., Phys. Rev. Lett. 15 (1980) 494; Europhysics News 13 (1982) 4, p. 2.

3. Bliek L., Melchert F., Warnecke P., Schlapp W., Weimann G., Ploog K., Ebert G. and Dorda G., Conf. on Prec. Electromagn. Meas. CPEM'84, Delft, Netherlands, to be published.

4. Niemeyer J., Hinken J. and Kautz R., Appl. Phys. Lett., to be published.

5. Kose V., PTB Mitt. 92 (1982) p. 249.

6. Bliek L., Braun E., Melchert F., Schlapp W., Warnecke P. and Weimann G., Metrologia 19 (1983) p. 83.

7. Cohen E.R. and Taylor B.N., H. Phys. Chem. Ref. Data 2 (1973) 663. 